\title{
Detection of Covid-19 Based on Chest Medical Imaging and Artificial Intelligent Techniques: A Review
}

\author{
Nawres Aref *, Hussain Kareem \\ Electrical Engineering Department, University of Technology, Baghdad, Iraq
}

Correspondence

*Nawres Aref

Electrical Engineering Department,

University of Technology, Baghdad, Iraq

Email: eee.19.18@grad.uotechnology.edu.iq

\begin{abstract}
Novel Coronavirus (Covid-2019), which first appeared in December 2019 in the Chinese city of Wuhan. It is spreading rapidly in most parts of the world and becoming a global epidemic. It is devastating, affecting public health, daily life, and the global economy. According to the statistics of the World Health Organization on August 11, the number of cases of coronavirus (Covid-2019) reached nearly 17 million, and the number of infections globally distributed among most European countries and most countries of the Asian continent, and the number of deaths from the Corona virus reached 700 thousand people around the world. . It is necessary to detect positive cases as soon as possible in order to prevent the spread of this epidemic and quickly treat infected patients. In this paper, the current literature on the methods used to detect Covid is presented. In these studies, the research that used different techniques of artificial intelligence to detect COVID-19 was reviewed as the convolutionary neural network (ResNet50, ResNet101, ResNet152, InceptionV3 and Inception-ResNetV2) were proposed for the identification of patients infected with coronavirus pneumonia using chest $X$-ray radiographs By using 5-fold cross validation, three separate binary classifications of four grades (COVID-19, normal (healthy), viral pneumonia and bacterial pneumonia) were introduced. It has been shown that the pre-trained ResNet50 model offers the highest classification performance (96.1 percent accuracy for Dataset-1, 99.5 percent accuracy for Dataset-2 and 99.7 percent accuracy for Dataset-2) based on the performance results obtained.
\end{abstract}

KEYWORDS: Covid-19, Artificial intelligent, K-mean, Segmentation.

\section{INTRODUCTION}

The recent emergence in China of the latest corona virus that triggered the 2019 corona virus disease (COVID-19) outbreak has posed significant public health threats worldwide (Wang et al., 2020). While transport to and from Wuhan and several other Chinese cities has been closed since 23 January 2020 [1]. In December 2019, the latest 2019 corona-virus (COVID-19) pandemic emerged in Wuhan, China and has become a significant global public health problem $[2,3]$. Severe acute respiratory syndrome corona-virus 2, also called SARS-CoV-2[4], is the virus that caused the COVID-19 pandemic disease. Coronaviruses $(\mathrm{CoV})$ are a broad family of viruses that cause diseases such as Middle East Respiratory Syndrome (MERS-CoV) and Extreme Acute Respiratory Syndrome arising from colds (SARS-CoV). Corona-virus disease (COVID-19) is a new species that has not been described previously in humans and was discovered in 2019. Corona-viruses are zoonotic, due to animal-to-human contamination [5]. Studies have shown that the SARS-CoV virus contaminates humans from musk cats and that the MERS-CoV virus contaminates humans from dromedaries $[5,6]$. Contamination of the COVID-19 virus from bats to humans is presumed [6]. The spread of the outbreak was rapidly triggered by respiratory transmission of the disease from person to person.

\section{A. Artificial Intelligent Techniques}

The goal of Artificial Intelligent (AI) is to ease human limitations. It gets perspective on human administration, packed by increasing restorative clinical data and the rapid development for informative strategies. This study summarizes current state of AI application in clinical administration while fighting COVID (19), the necessity of using artificial intelligent in fighting the COVID (19) crisis. In addition, while understanding this virus, the high light of the big data application. It also identifies various intelligent methods that can be applied to different kinds of pandemics based on medical information. In clinical data analysis, we identify the current AI techniques, including neural system, classical SVM, and important learning. Also, in combating various related viruses to COVID (19), Emphasis was placed on regions that use Artificial Intelligent oriented cloud computing. This study is an experiment in helping

This is an open access article under the terms of the Creative Commons Attribution License, which permits use, distribution and reproduction in any medium, provided the original work is properly cited.

(C) 2021 The Authors. Published by Iraqi Journal for Electrical and Electronic Engineering by College of Engineering, University of Basrah. 
medical researchers solve the problems they face when processing treating big data with COVID (19) with a precision of up to 90 percent, the investigated approaches have made advances in medical data analysis. It also end up with comprehensive discussion about how the implementation of AI can be a big advantage in the fight against many related viruses [7]. Artificial intelligent (AI) excitement has been strong for as far back as hardly any years, hitting the top in 2018. This promotion is due to the crucial advances in the field of AI and, in fact, broad application [8]. AI steps have sent tremendous waves across restorative administration, fueling a working discussion as to whether AI specialists can later replace human experts as time goes by. Artificial intelligence helps authorities determine the best medical options or replaces human judgment in a specific field that has a general advantage, such as radiology. However, with the general development of information and the active improvement of information, expressive methods have made the profitable advanced uses of this AI technology possible. Inferred with clinical requests, basic AI strategies can unlock clinical data, which along these lines effectively lead $[9,10]$. Affirmed cases corona-virus COVID-19 infection outweigh those of serious respiratory disorder (such as SARS). By link, the death case confirm by SARS in 2003 was 774 individuals. COVID -19 and SARS both are spreading over land areas, taint animals, individuals, use comparative mechanics to penetrate and contaminate cell [11].

Physical evaluation jots and outcomes from the medical process center are also identified as part of other essential fields of knowledge as shown in Fig. (1). It also identifies areas or fields where the implementation of AI in recent years has been of great interest. They are recognized with an image, even inherited and essentially electro-physiological (EP) data, just because they consist of big parts of unstructured paper writings that are not good for study, such as medical jots. As a result, the comparative AI application center first switches to machine-justifiable electronic medical records over disorganized content (EMR) [12-15]. For example, however, Karakülah G, Dicle O (2020) [16] used AI advances to eliminate phenotypic highlights from case reports to enhance the accuracy of finding inborn abnormalities. Becky McCall (2020) [17] presented a features that the application for AI by Infer vision restricts the weight of the procedures by facilitating COVID-19 decisions and observations. The measurement learns and increases accuracy along with the infection when an ever-increasing number of sweeps is performed. By decreasing the weight of clinicians in a case, such as present COVID(19) episode, the estimate of AI is become integral factor and draws attention to the rapid development of the current outbreak of contamination of medical service experts, as the introduction of Infervision AI will help ensure practitioners. The condition of clinicians on the cutting edge is characterized by the passing of clinical experts, such as the doctor who detected the infection. As well as describes clinic-related transmission from one man to another, accounting for $41 \%$ of the current cases in a study of patients at Wuhan Hospital. More than 1,000 emergency clinic practitioners have been confirmed to be infected. As this is the position that Infervision AI will support. Artificial intelligent means the rapid discrimination of COVID-19 pneumonia infections that can be visualized through a lung CT scan, to measure its capacity, shape and thickness, and to examine the change in images of various lung ulcers, and this provides all the quantitative reports that help in making a quick decision for specialists.. This can take as long as 15 minutes when manually reading the CT output, whereas AI can wrap up the image in no more than 10 seconds. The implementation of this COVID-19 invention is not yet distributed in a diary inspected by a companion.

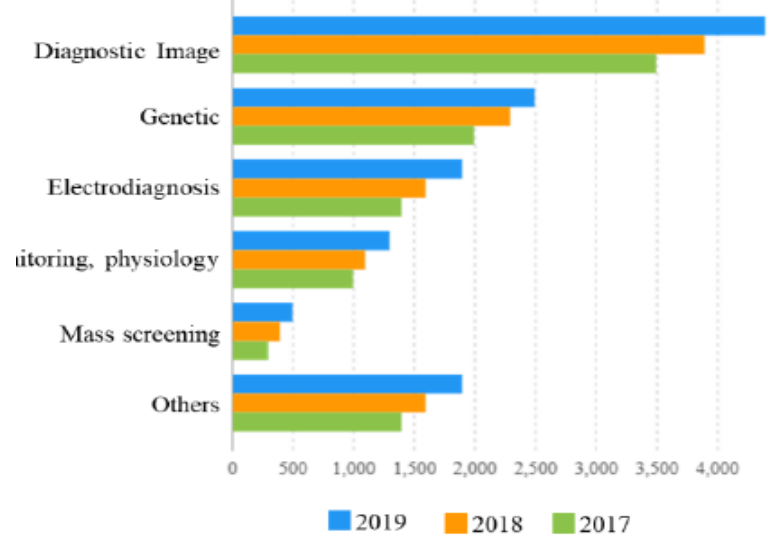

Fig. 1 Data types Considered in AI Concerning Covid-19.

\section{B. Artificial Intelligent Learning Techniques}

A big warning from clinical experts is that the conversation has nothing to do kindly with AI they were conducting, which has a more subtle, clearer meaning, was kind of from a media point of view of AI. In their examination, they had all the conclusions to be confident of the improvement, lamenting the too-moderate interpretation from analysis rehearsal regardless for whether collaborating with social insurance industry was seen as an approach to speed up the interpretation. In addition, they griped on problems created by enactment with regard to gathering examination details. As they indicated, this was the best way to increase their examination funding and enable good improvement of $\mathrm{AI}$ and assurance of its quality along these lines. In the dialogue about the issues in general, they did not take an interest and focused on their analysis approach. One of the experts a specialist in air transport who spoke about human-PC contact (HCI). Nevertheless, for him, while HCI is not unique for $\mathrm{AI}$, scrutinizing the robotization of orders and cut-off points should be seen as one of the key goals of the combination of AI into social insurance systems. He explained that the primary thoughts, right now, are to designate it only when it's simple. It is a danger of deskilling without this. Deskilling is when the professional who does not have foggiest idea of how to do assignment he did before loses the opportunity because he stopped doing it to help the computer. Below, the associated AI categorized learning methods will be listed [18].

1) Supervised Learning

This is one of the health system's most used techniques and it is typically ingrained. In making specific desires, this learning method uses data and learn mapping between yield 
and its related input while collecting analysis through learning strategy to recognize items that depend on close characteristics. Actually, different ways of imagining an outcome or the potential outcome or collecting large number for necessary states [19] are used. Currently, the SVM, NN, and RC are the standard procedures performed correctly. The limit is normally defined in the medical system when setting up these calculations. The best relation between yield and data can be induced cost job tell practitioner how far it can be from exact output by then, so this goes around as data signal. The model had option of arranging medical data with an accuracy of up to $90 \%$. All things considered substantial learning; right now, in all places, it has become a leap forward system. An astute stage for programmed observation and prediction of COVID -19- can be assembled by supervised learning. It is also possible to create a neural system to remove the visual features of this disease, and this would aid in the legitimate control and treatment of affected individuals. It can provide most recent patient updates and respond with associated queries with COVID-19. By considering contribution layer [20]. A learning approach can encourage systems setting. An Xception, CNN approach based on the convolution of distinguishable layer was Also using two layer of convolution, trailed with layers of convolution that are divisible in depth, four layers of convolution, and completely related layer[21]. Whereas it is used to classify the bed posture using different bed pressure sensors in [22]. It can serve as a useful tool in combating COVID -19 with its capability and good efficiency result.

\section{2) Unsupervised Learning}

Learning technology does not use information or names in place of the above learning method which uses specific data. In finding the covered structures for the given data, this method is widely used and it divides it into close groups. It is generally used to elucidate evidence of display and design distinction. This is a promising kind of estimate to satisfy the general need for AI, but it is not absent in the slightest degree, such as the above-discussed learning technique. The most commonly recognized unsupervised technique is $\mathrm{K}$-means, and also the auto encoder. One of the most common roles for this teaching technique in the medical field is recognition of exoticism [23]. If there is some kind of intrusion or any counterfeit read from the affiliation data, the data made from the affiliation will start from equal scattering, this information will be hailed an exception and can be hailed or seen without a lot of stretch. A common and widely used to group mechanism in image processing [24] is the K-means. This calculation allows the client to specify the sum of the k cluster to be generated. It was used to isolate clamor from PD flags, as in [24], with no attention being given to the few subcategories imaginable within PD conferences and disruption. With this term, it can thus be used for CT images and other medical applications, including COVID (19). In [25], at learning model of opportunistic cameras, the author put forward a new framework that capture moving data from the stream. The neural system is subsequently using with foresee the movement of the streaming event. This movements are used to attempt and evacuate any obscured movement in stream images.

\section{3) Deep Learning in Covid-19}

To perceive, interpret, predict, and give an explanation for the COVID (19) infection, use of deep getting to know can be really helpful and aid preserve financial effects. There has been a scramble when you consider that the outbreak of the pandemic to use and study AI and distinctive information-analytical strategies for these reasons [26]. It pastimes to draw a shortly take-away from a rapidly evolving discussion, create a sequence of large studies, medical arrangements, and clinical examination facts repositories. In addition to existence and monetary damage, the rate of the COVID-19 pandemic will be awful if deep reading is now not updated or later. It is of best undertaking presently to develop AI, one of the most inspiring information-analytical tools, to help limit these vulnerabilities. Encouragingly, the title was as soon as spoke returned to with the aid of the use of archives researchers. AI has now no longer been successful in opposition to COVID-19 yet, however. It is of biggest interest nowadays to strengthen AI, one of the most inspiring information-analytical tools, to assist limit these vulnerabilities. Encouragingly, the name used to be replied to by way of statistics researchers. AI has no longer been worthwhile against COVID-19 yet, however. It is not going that, at some stage in the modern-day pandemic, these troubles fail to be of exceptional benefit. Rather, the pandemic can help with deep learning. It can also additionally be critical to accumulate empirical facts about who is irresistible because of policies to retailer lives and ward off financial ruin. As well as advocated the use of deep studying to comply with and simulate how the sickness of COVID-19 would spread over time and space. For example, a effective neural machine was once created to predict its spread after a previous pandemic, such as the zika infection[26]. For instance, models be re-prepared the use of records from the COVID-19 pandemic. This is going on now, by all accounts. They proposed the utilization of calculations organized to count on the occasional fluidity at a range of institutions, which are currently being re-prepared on the groundwork of new COVID-19 details. Authors that via classifying pneumonia as abnormal, the deep analyzing system can without difficulty and accurately identify chest X-rays from Covid-19 patients, which may also help speed up look up in the midst of a progressing pandemic. Nonetheless, 87 percentage of the time, according to health offerings professionals, these gaining knowledge of structures have effectively diagnosed the illness. Additionally, such frameworks are proper ninety two proportion of the time, as a substitute than ninety share like human experts [27].

\section{Commuting AI TechniQues IN COMBating CovID-19}

In contemporary years, simulation planning has swiftly grown for the duration of the globe. This is relatively related to the ordered residency coaching scheme for clinical practitioner that commenced in 2015. In any event, the key goal of these re-enactments used to be once to fulfill the instructive desires of the particular preparatory applications 
or to red meat up these academic applications [28]. Recreation has flip out to be an tremendous system fighting in the direction of the virus in the aftermath of grasp the want for training, as it can guarantee quiet protection as top as supply a sheltered gaining expertise of and practice surroundings for HCWs to take care of the COVID-19[29]. The simulation and forecasting of the SARS-CoV-2 virus, Boltzmann's function-based regression evaluation used to be proposed. This strategy used to be applied to the mixed cases examined in Wuhan Province. A direct estimation of potential said cases is furnished via the Boltzmann function. It would no longer choose an amazing deal higher distinctive data for analysis, in addition to the Boltzmann attribute [30]. Only cumulative cases are needed. It also made it possible in other cities to predict potential courses. However, due to uncertainty in the data being published, predictions of the outcomes are not guaranteed [31,32]. When use five pre-trained models based on the convolutionary neural network (ResNet50, ResNet101, ResNet152, InceptionV3 and Inception-ResNetV2) were proposed for the identification of patients infected with coronavirus pneumonia using chest X-ray radiographs By using 5-fold cross validation, three separate binary classifications of four grades (COVID-19, normal (healthy), viral pneumonia and bacterial pneumonia) were introduced. It has been shown that the pre-trained ResNet50 model offers the highest classification performance (96.1 percent accuracy for Dataset-1, 99.5 percent accuracy for Dataset-2 and 99.7 percent accuracy for Dataset-2) based on the performance results obtained [33]. Fig. (2) illustrated a number of papers of Technique Covid19, Fig. (3) show a percentage of accuracy with Techniques applied to Detection COVID-19.Many researches study a COVID-19 are summarized in Table (1) [33-42] .Other researches applied AI for detection other diseases lung cancer [43] and using a other types of segmentation, De-nosing filtering of image with a techniques of feature extraction and Classification [44-47].

\section{No. of Papers}

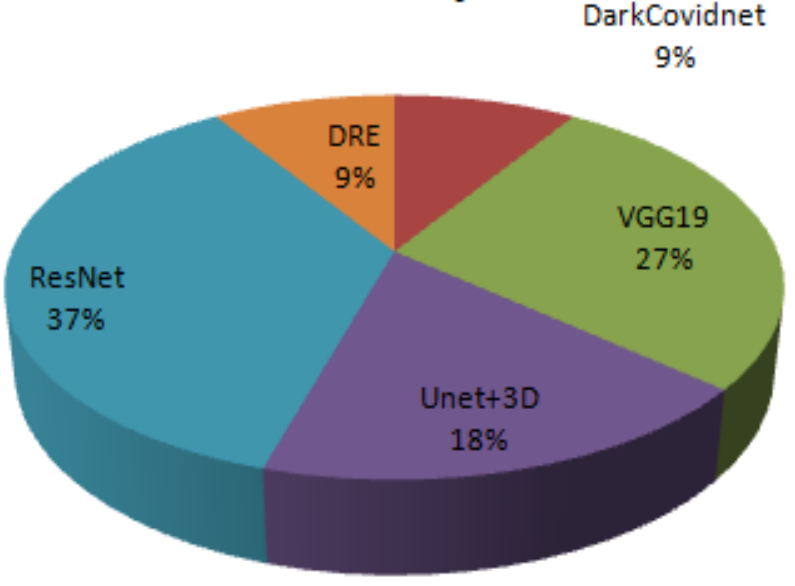

Fig. 2 Number of papers of Technique Covid19.

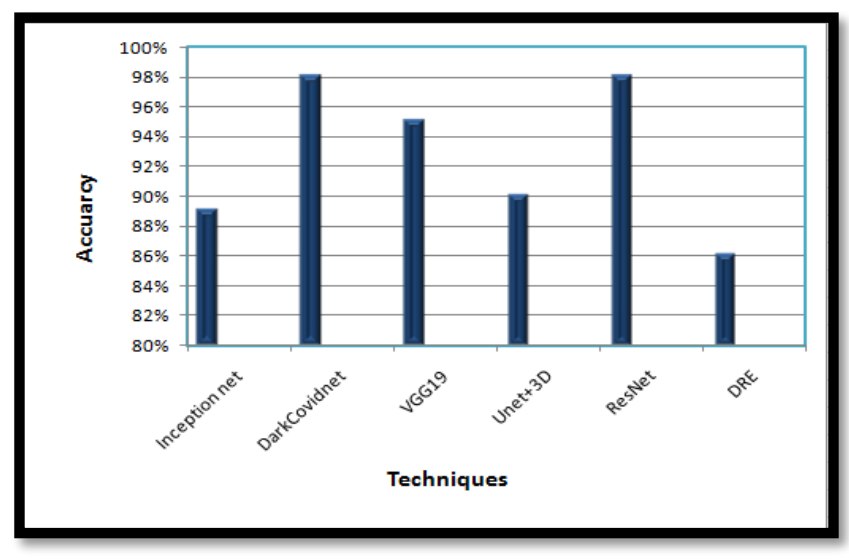

Fig.3 Accuracy of Techniques

TABLE (I)

Summary of previous studies to detect Covid19

\begin{tabular}{|c|c|c|c|c|}
\hline Authors & $\begin{array}{l}\text { Type of } \\
\text { Images }\end{array}$ & Number of Cases & $\begin{array}{l}\text { Method } \\
\text { Used }\end{array}$ & $\begin{array}{c}\text { Accuracy } \\
(\%)\end{array}$ \\
\hline Narin et al.[33] & Chest X-ray & $\begin{array}{l}50 \text { Covid-19(+) } \\
50 \text { Covid- } 19(-)\end{array}$ & $\begin{array}{l}\text { Deep CNN } \\
\text { ResNet-50 }\end{array}$ & 98 \\
\hline Zheng et al.[34] & Chest CT & $\begin{array}{c}313 \text { Covid- } 19(+) 229 \text { Covid- } \\
19(-)\end{array}$ & $\begin{array}{l}\text { UNet+3D } \\
\text { Deep } \\
\text { Network }\end{array}$ & 90.8 \\
\hline Sethy and Behra[35] & Chest X-ray & $\begin{array}{c}25 \text { Covid-19 (+) } 25 \text { Covid-19 } \\
(-)\end{array}$ & $\begin{array}{c}\text { ResNet50+ } \\
\text { SVM }\end{array}$ & 95.38 \\
\hline Ying et al. [36] & Chest CT & $\begin{array}{c}777 \text { Covid- } 19(+) \\
708 \text { Healthy }\end{array}$ & DRE-Net & 86 \\
\hline Hemdan et al_[37] & X-ray images & $\begin{array}{c}25-\text { Covid-19 } \\
25-\text { No-Findings }\end{array}$ & $\begin{array}{c}\text { VGG19, } \\
\text { DenseNet12 } \\
1\end{array}$ & 90.00 \\
\hline $\mathrm{Xu}$ et al.[38] & Chest CT & $\begin{array}{c}219 \text { Covid-19 (+) } \\
224 \text { Viral Pneumonia } 175 \\
\text { Healthy }\end{array}$ & $\begin{array}{l}\text { ResNet + } \\
\text { Location } \\
\text { Attention }\end{array}$ & 86.7 \\
\hline Ozturk et al_[39] & X-ray images & $\begin{array}{l}127 \text {-Covid-19, } \\
500 \text { - No-Findings }\end{array}$ & $\begin{array}{c}\text { DarkCovidN } \\
\text { et }\end{array}$ & 98.08 \\
\hline $\begin{array}{l}\text { Apostolopoulos et } \\
\text { al. [40] }\end{array}$ & X-ray images & $\begin{array}{l}224-\text { Covid-19, } \\
504-\text { No-Findings }\end{array}$ & VGG19 & 98.75 \\
\hline Ioannis et al.[41] & Chest X-ray & $\begin{array}{l}224 \text { COVID-19 (b) } 700 \\
\text { Pneumonia } 504 \text { Healthy }\end{array}$ & VGG-19 & 93.48 \\
\hline Wang et al. [42] & CT images & $\begin{array}{c}325 \text { - Covid-19 } \\
740 \text { - Pneumonia }\end{array}$ & InceptionNet & 89.50 \\
\hline
\end{tabular}

\section{RESULTS AND DISCUSSION}

First stage to detect COVID-19 doing preprocessing stage so, de noising of images and edit contrast. The second stages is segmentation that cut an image to parts and cut a inject region as well as a third stages is feature extraction that determine specification of cut parts ,Finally at last stages is classification type regional segmentation method and classification FFBP that determine an image is inject of COVID-19 or normal stages as shown in Fig.(4) and Fig.(5) respectively, this results selected from 300 person in normal and COVID-19 inject then applied in MATLAB(2018a) । m-file as shown in Fig. (4) and Fig. (5) when insert images of normal or inject and a code will give a state of detection is inject or not.

It is just the beginning to implement AI strategies on COVID-19 quest. There have been attempts to add AI to the full COVID-19 imaging-based prognosis pipeline. Nevertheless, there are still many things to be carried out in 
the future. Workflows for AI-empowered image acquisition have confirmed that the scanning process is no longer just more effective, but also fantastic in defending scientific personnel from COVID-19 infection. Looking forward, additional AI-enabled uses are planned to be incorporated into the workflow of image acquisition to encourage higher first-class scanning and decreased patient-fed radiation dose. For example, in order to achieve the greatest image efficiency, higher specific AI-based automatic ISO-centering and scan willpower varies. In addition, X-ray exposure parameters can be measured and optimized robotically for the patient's AI inferred body region thickness, ensuring that only the right amount of radiation is used throughout the scan, which is especially important for low-dose imaging. In the early stage of the disease, medical pixels normally display weak radiological signs, and so learning about this stage is necessary to help with the ambiguity of scientific diagnosis. In the meantime, many existing AI segmentation and prognosis research are focused on limited samples, which can also lead to findings becoming overwhelming. To render the findings clinically advantageous.

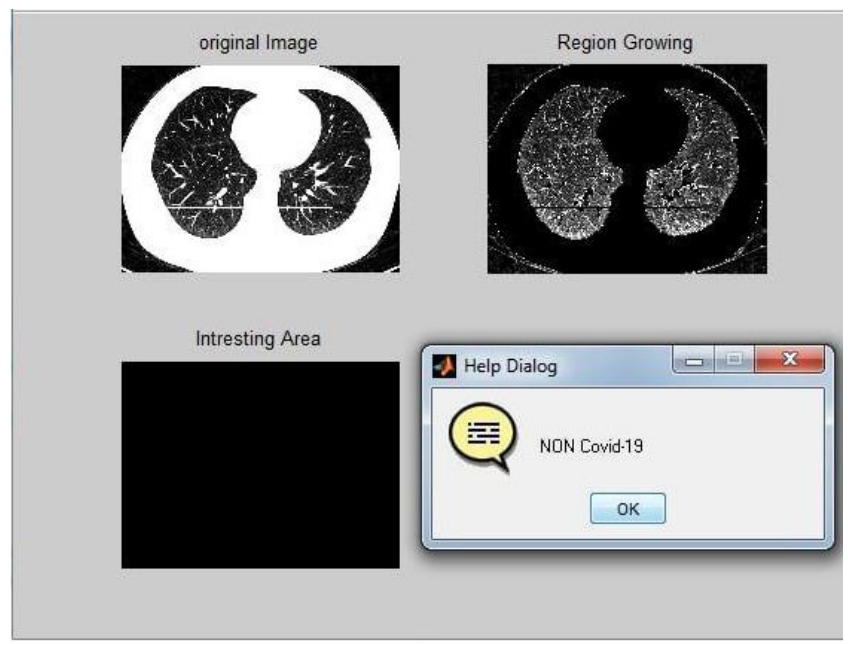

Fig. 4 Detection Normal State

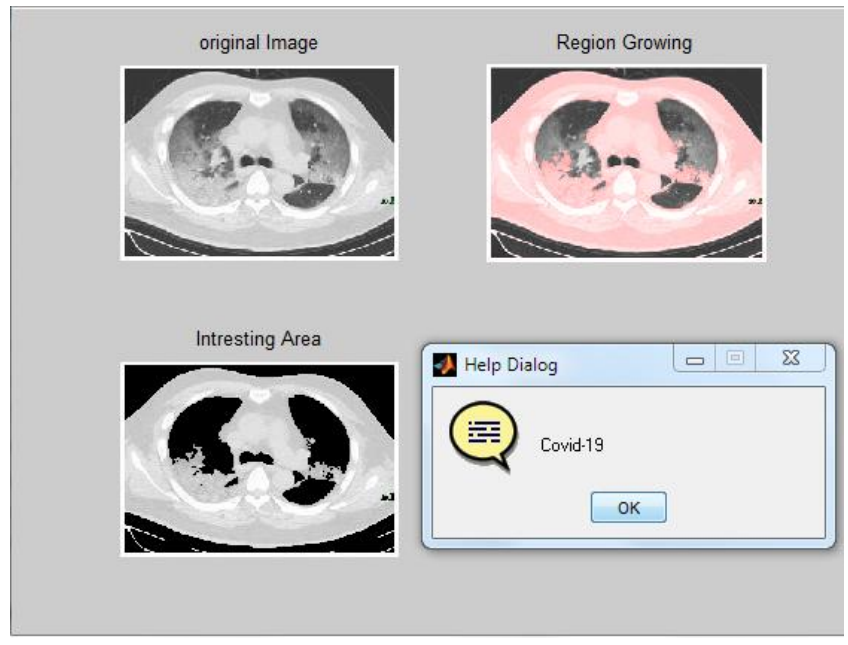

Fig. 5 Detection COVID19 State

\section{CONCLUSION}

COVID-19 is a disease that has spread across the world. In contrast to COVID-19, intelligent clinical imaging performed a fundamental role in the battle. This paper explores how AI in COVID-19 applications provides healthy, accurate and environmentally friendly imaging choices. Clever imaging systems, scientific diagnosis, and innovative science are analyzed in depth, covering the entire pipeline of AI-enabled imaging capabilities in COVID-19. To demonstrate the efficacy of AI-empowered scientific imaging for COVID-19, two imaging modalities, i.e., X-ray and CT, are used. It is definitely well worth noting that with COVID-19, imaging only provides partial data regarding victims. In order to resource broader COVID-19 image, identification and diagnosis, it is also important to combine imaging documents with both scientific manifestations and laboratory review penalties. Fusing facts from these multi-source results, AI can demonstrate its herbal functionality to perform correct and environmentally pleasant diagnosis and evaluation. the convolutionary neural network (ResNet50, ResNet101, ResNet152, InceptionV3 and Inception-ResNetV2) were proposed for the identification of patients infected with coronavirus pneumonia using chest X-ray radiographs By using 5-fold cross validation, three separate binary classifications of four grades (COVID-19, normal (healthy), viral pneumonia and bacterial pneumonia) were introduced. It has been shown that the pre-trained ResNet50 model offers the highest classification performance (96.1 percent accuracy for Dataset-1, 99.5 percent accuracy for Dataset-2 and 99.7 percent accuracy for Dataset-2) based on the performance results obtained.

\section{CONFLICT OF INTEREST}

The authors have no conflict of relevant interest to this article.

\section{REFERENCES}

[1] T. Ji, Zhenwei Liu, G. Wang, Xu. Guo, S. Akbar khan, Changchun Lai, H. Chen, S. Huang, S. Xia, Bo Chen, Hongyun Jia, Y. Chen and Qiang Zhou, "Detection of COVID-19: A review of the current literature and future perspectives", Biosensors and Bioelectronics, Vol. $166,2020$.

[2] K. Roosa, Lee, Y., Luo, R., Kirpich, A., Rothenberg, R., Hyman, J. M., Yan, P., and Chowell, G., "Real-time forecasts of the COVID-19 epidemic in China from February 5th to February 24th, 2020", Infectious Disease Modelling, Vol. 5, pp. 256-263, 2020.

[3] Yan, L., Zhang, H. T., Xiao, Y., Wang, M., Guo, Y., Sun, C., Tang, X., Jing, L., Li, S., Zhang, M., Xiao, Y., Cao, H., Chen, Y., Ren, T., Jin, J., Wang, F., Xiao, Y., Huang, S., Tan, X., Huang, N., Jiao, B., Zhang, Y., Luo, A., Cao, Z., $\mathrm{Xu}, \mathrm{H}$., and Yuan, Y., "Prediction of criticality in patients with severe Covid-19 infection using three clinical features: a machine learning-based prognostic model with clinical data in Wuhan", medRxiv, 2020. 
[4] S. B. Stoecklin, P. Rolland, Y. Silue, A. Mailles, C. Campese, A. Simondon, M. Mechain, L. Meurice, M. Nguyen, C. Bassi, , E. Yamani, , S. Behillil, S. Ismael, D., Malvy, D.Nguyen, F. Lescure, X., Georges, S., Lazarus, C., Tabaï, A., Stempfelet, M., Enouf, V., Coignard, B., Levy-Bruhl, D. and Team, I., "First cases of coronavirus disease (COVID-19) in France: surveillance, investigations and control measures", Eurosurveillance, Vol. 25, No. 6, 2020.

[5] Coronavirus. World Health Organization: (2020) https://www.who.int/health-topics/coronavirus.

[6] C. Huang, Y. Wang, Li, X., Ren, L., Zhao, J., Hu, Y., Zhang, L., Fan, G., Xu, J., Gu, X., Cheng, Z., Yu, T., Xia, J., Wei, Y., Wu, W., Xie, X., Yin, W., Li, H., Liu, M., Xiao, Y., Gao, H., Guo, L., Xie, J., Wang, G., Jiang, R., Gao, Z., Jin Q., Wang, J., and Cao, B., "Clinical features of patients infected with 2019 novel coronavirus in Wuhan, China.”, The Lancet, Vol. 395, No. 10223, pp. 497-506, 2020.

[7] A. Adedoyin Hussain, O. Bouachir, F. Al-Turjman and M. Aloqaily, "AI Techniques for COVID-19", IEEE Access, 2020.

[8] TB. Murdoch and AS. Detsky, "The inevitable application of big data to health care", JAMA, Vol. 309, PP. 1351-2, 2013.

[9] Kolker E, Özdemir V, and Kolker E. (2016) "How Healthcare can refocus on its Super-Customers (Patients, $\mathrm{n}=1$ ) and Customers (Doctors and Nurses)", by Leveraging Lessons from Amazon, Uber, and Watson. OMICS 20, pp. 329-33.

[10] Dilsizian SE and Siegel EL., "Artificial intelligence in medicine and cardiac imaging: harnessing big data and advanced computing to provide personalized medical diagnosis and treatment", Curr Cardiol Rep, Vol. 16, No. 441, 2014.

[11] Xu X, P. Chen, and J. Wang, "Evolution of the novel coronavirus from the ongoing Wuhan outbreak and modeling of its spike protein for risk of human transmission", Sci China Life Sci, 2020, DOI: 10.1007/s11427-020-1637-5.

[12] Administration UFaD. Guidance for industry: electronic source data in clinical investigations, 2020. [Online] Available: https://www. fda. gov/ downloads/drugs/ guidances/ ucm328691.

[13] RJ. Gillies, PE. Kinahan, Hrica and H. Radiomics, "Images are more than pictures, they are data", Radiology, Vol. 278, No. 2, pp. 563-77, 2016.

[14] Li. CY, GY. Liang and Yao WZ., "Integrated analysis of long noncoding RNA competing interactions reveals the potential role in progression of human gastric Cancer", Int J Oncol Vol. 48, pp. 1965-76, 2016.

[15] Shin H, Kim KH and Song C., "Electro-diagnosis support system for localizing neural injury in an upper limb", J Am Med Inform Assoc, 2016.

[16] G. Karakülah, O. Dicle and Koşaner O., "Computer based extraction of phenotypic features of human congenital anomalies from the digital literature with natural language processing techniques", Stud Health Technology Inform, Vol. 205, pp. 570-4, 2014.
[17] Becky McCall., "COVID-19 and artificial intelligence: protecting health-care workers and curbing the spread", Published by Elsevier Ltd, 2020. Doi: 10.1016, S2589-7500(20)30054-6.

[18] Commissioner of the Statement from FDA Commissioner Scott Gottlieb, M.D. on steps toward a new, tailored review framework for artificial intelligence-based medical devices. FDA. (2019) [Online] Available:

https://news-events/press-announcements/statement-fdacommissioner-scott-gottlieb-mdsteps.

[19] Al-Turjman F. Intelligence and security in big 5G-oriented IoNT: An overview, Elsevier Future Generation Computer Systems, Vol. 102, pp. 357-368, 2020.

[20] J. Bedford, D. Enria, J. Giesecke, D.L. Heymann, C. Ihekweazu, V. Kobinger, Lane H. C, Memish V, Oh M.D and Schuchat A., "COVID-19: towards controlling of a pandemic", Lancet, Vol. 395, pp. 1015-1018, 2020.

[21] F. Chollet, "Xception: deep learning with depthwise separable convolutions", in: Proceedings of the IEEE Conference on Computer Vision and Pattern Recognition, pp. 1251-1258, 2017.

[22] M. G, Lina J.-M, and G. Kaddoum, "Artificial neural network for in-bed posture classification using bed-sheet pressure sensors", IEEE Journal of biomedical and health informatics, no. 10, pp. 101-110, 2020.

[23]Gozes O, Frid-Adar M, Greenspan H, Browning PD, Zhang H, Ji W, Bernheim A and Siegel E. Rapid, "AI development cycle for the Coronavirus (COVID-19) pandemic: initial results for automated detection \& patient monitoring using deep learning CT image analysis", arXiv preprint arXiv:05037, 2020.

[24] A. Ranjan, V. Jampani, L. Balles, K. Kim, D. Sun, J. Wulff, and M. J Black, "Competitive collaboration: Joint unsupervised learning of depth, camera motion, optical flow and motion segmentation". In Proceedings of the IEEE Conference on Computer Vision and Pattern Recognition, pp. 12240-12249, 2019.

[25] A. Zihao Zhu, L. Yuan, K. Chaney, and K. Daniilidis, "Unsupervised event-based learning of optical flow, depth, and egomotion", In Proceedings of the IEEE Conference on Computer Vision and Pattern Recognition, pp. 989-997, 2019.

[26] W. Naude, "Artificial Intelligence against COVID-19": An Early Review, Vol. 23, No. 4, 2020 [Online] Available:

https://towardsdatascience.com/artificial-intelligence-aga inst-covid-19-an-early-review-92a8360edaba.

[27] M. Smolaks, "Deep learning systems can speed up diagnosis of Covid-19 patients", 2020. Available: https://aibusiness.com/deep-learning-systems-can-identif y-covid-19-infections-on-x-rays.

[28] Sh. Wang, Bo Kang, Jinlu Ma, Xianjun Zeng, Mingming Xiao, Jia Guo, Mengjiao Cai, Jingyi Yang, Yaodong Li, Xiangfei Meng and Bo Xu., "A deep learning algorithm using CT images to screen for Corona Virus Disease (COVID-19)", MedRxiv preprint, 2020. Doi: https://doi.org/10.1101/2020.02.14.20023028. 
[29] A. Devereaux, MD. Christian and JR. Dichter, "Summary of suggestions from the task force for mass critical care Summit", Chest, Vol. 133, 2018. Doi:10.1378/chest.08-0649.

[30] N.E. Huang, and F. Qiao, "A data driven time dependent transmission rate for tracking an epidemic: a case 305 study of 2019-nCoV", Science Bulletin, 2019. [Online] Available: https://doi.org/10.1016/j.scib.02.005. 2019

[31] Y. Yang, "Epidemiological and clinical features of the 2019 novel coronavirus outbreak in China", medRxiv, 2020. https://doi.org/10.1101/2020.02.10.20021675.

[32] Wu, J.T., K. Leung, and G.M. Leung, "Now casting and forecasting the potential domestic and international 311 spread of the 2019-nCoV outbreak originating in Wuhan, China", a modelling study. Lancet, 2020. Doi: 312 10.1016/S0140-6736 (20)30260-9.

[33] A. Narin, C. Kaya, and Pamuk, Z., "Automatic detection of coronavirus disease (COVID-19) using X-ray images and deep convolutional neural networks", arXiv, 2020.

[34] C. Zheng, X. Deng, Fu, Q., Zhou, Q., Feng, J., et al., "Deep learning-based detection for COVID-19 from chest CT using weak label", medRxiv, 2020. DOI: https://doi.org/10.1101/.03.12.20027185.

[35] P.K. Sethy, S.K. Behera, "Detection of Coronavirus Disease (COVID-19) Based on Deep Features", vol.1, reprints, 2020 (doi:10.20944/preprints202003.0300.v1)

[36] Y. Song, S. Zheng, L. Li, X. Zhang, X. Zhang, Z. Huang, Y. Chong, "Deep learning enables accurate diagnosis of novel coronavirus (COVID-19) with CT images", medRxiv, 2020.

[37] E.E.D. Hemdan, M.A. Shouman, M.E. Karar, "COVIDX-Net: A Framework of Deep Learning Classifiers to Diagnose COVID-19 in X-Ray Images", arXiv preprint, 2020.

[38] X. Xu, X. Jiang, C. Ma, P. Du, X. Li, S. Lv, et al., "Deep Learning System to Screen Coronavirus Disease 2019 Pneumonia", arXiv preprint, 2020.

[39] T. Ozturk, M. Talo, EA. Yildirim, UB. Baloglu, Yildirim O, Acharya R., "Automated detection of COVID-19 cases using deep neural networks with X-ray images", Comput. Biol. Med., Vol. 121, 2020. https://doi.org/10.1016/j.compbiomed. 103792.

[40] Apostolopoulos ID, Mpesiana TA., "COVID-19: automatic detection from $\mathrm{x}$-ray images utilizing transfer learning with convolutional neural networks", Physical and Engineering Sciences in Medicine, Vol. 43, pp.635-40, 2020. https://doi.org/10. 1007/s13246- 02000865- 4, 2020.

[41] Ioannis D. Apostolopoulos1, Tzani Bessiana, "COVID-19: Automatic Detection from X-Ray Images Utilizing Transfer Learning with Convolutional Neural Networks", arXiv:11617, 2003.

[42] S. Wang, B. Kang, J. Ma, X. Zeng, M. Xiao, J. Guo, B. $\mathrm{Xu}$, " A deep learning algorithm using $\mathrm{CT}$ images to screen for Corona Virus Disease (COVID-19)", medRxiv, 2020.
[43] I. S. Abed, "Lung Cancer Detection from X-ray images by combined Backpropagation Neural Network and PCA", Engineering and Technology Journal, Vol. 37, Part A, No. 05, pp. 166-171, 2019.

[44] A..S. Yaseen, R. S. Zamel and J. H. Khlaief,(2021), "Wavelet-Based Denoising Of Images," Engineering and Technology Journal, Vol. 37, Part B, No. 2, pp. 54-60, 2019.

[45] H. M. Ahmed, and M. A. Taha, "A Brief Survey on Modern Iris Feature Extraction Methods", Engineering and Technology Journal, Vol. 39,Part A, No. 01, pp. 123-129, 2021.

[46] H. .N. Abdullah and N.H. Abdulghafoor, "Automatic objects detection and tracking using FPCP, Blob analysis and kalman filter", Engineering and Technology Journal, Vol. 38, No. 02, pp. 246-254, 2020.

[47] H.A. Jeiad, "Indian Number Handwriting Features Extraction and Classification using Multi-Class SVM", Engineering and Technology Journal, Vol. 36, No. 1, pp. 33-40, 2018. 